\title{
Curcumin suppresses stem-like traits of lung cancer cells via inhibiting the JAK2/STAT3 signaling pathway
}

\author{
LICHUAN WU $^{1 *}$, LIANGXIA GUO $^{1 *}$, YUEHUI LIANG $^{1}$, XU LIU $^{1}$, LIHE JIANG $^{1,2}$ and LISHENG WANG ${ }^{1}$ \\ ${ }^{1}$ School of Chemistry and Chemical Engineering, Guangxi University, Nanning, Guangxi 530004, P.R. China; \\ ${ }^{2}$ Tennessee Center for Botanical Medicine Research, Department of Biology, \\ Middle Tennessee State University, Murfreesboro, TN 37132, USA
}

Received June 11, 2015; Accepted August 10, 2015

DOI: $10.3892 /$ or.2015.4279

\begin{abstract}
Tumor recurrence and drug resistance are the main obstacles blocking effective treatment of cancer patients. Cancer stem cells (CSCs) have been demonstrated to be highly related to tumor recurrence and drug resistance. Thus, eliminating CSCs may be an alternative for cancer therapy. Tumor sphere formation is a functional assay to enrich the CSC-like cells. In the present study, we tested the effects of curcumin on lung cancer stem-like cells and report that in addition to inhibition on the proliferation and colony formation of lung cancer cells, curcumin reduces tumor spheres of H460 cells. Moreover, by molecular docking analysis and tumor sphere assay we discover that curcumin was able to inhibit JAK2 activity and reduce tumor spheres via inhibiting the JAK2/STAT3 signaling pathway. In a lung cancer xenograft nude mouse model, curcumin strongly repressed tumor growth. These results imply curcumin may be a potential drug in lung CSC elimination and cancer therapy.
\end{abstract}

\section{Introduction}

Lung cancer is the most deadly malignant tumor (1). There are two major types, non-small cell lung cancer (NSCLC) and small-cell lung cancer (SCLC) which comprise 85 and $15 \%$ of all cases, respectively. Tumor-initiating cells, also termed cancer stem cells (CSCs), are a rare population of cancer cells which possess the ability to self-renew and differentiate (2). CSCs were first isolated in 1997 in leukemia (3). Subsequently, CSCs have been identified in solid tumors, including breast (4), brain $(5)$, prostate $(6)$, melanoma $(7)$, colon $(8,9)$ and lung

Correspondence to: Professor Lisheng Wang, School of Chemistry and Chemical Engineering, Guangxi University, Daxue East Road, 100 Nanning, Guangxi 530004, P.R. China

E-mail:wmmglatw@126.com

\section{*Contributed equally}

Key words: curcumin, tumor sphere, JAK2-STAT3, lung cancer, cancer stem cell cancer (10,11). Since the isolation of CSCs in 1997, a growing body of literature has demonstrated that tumor initiation, recurrence and drug resistance are highly related to CSCs (12-14). Thus, CSCs is likely a plausible target for cancer therapy (15). CSCs were identified using flow cytometry-based cell sorting of specific surface markers or tumor sphere forming assay. Tumor spheres, which could reflect tumor cell stem-like property, are multicellular three-dimensional clones enriching CSCs (2,3). Suppressing tumor spheres may impair cancer cell stem-like property.

The JAK2/STAT3 pathway mediates the effects of a spectrum of cytokines and growth factors. It can be transiently activated in normal cells upon stimulation while in cancer cells, it is constitutively active $(16,17)$. JAK2/STAT3 signaling pathway plays a crucial role in various cancer initiation and development stages, and active STAT3 is commonly associated with a worse prognosis (18-21). It is also reported that JAK2/STAT3 signaling pathway is involved in CSCs in many malignant, including lung CSCs and aberrant expression of JAK2/STAT3 signaling pathway in CSCs can promote cancer initiation (22-24). Thus, JAK2/STAT3 pathway could be a targetable pathway for CSC elimination.

Curcumin (diferuloylmethane, Fig. 1A), a phenolic compound isolated from the plant Curcuma longa, has been used in traditional medicines in China for thousands of years. It exhibits anticancer effects by induction of growth inhibition, cell cycle arrest and apoptosis in various types of cancer (25-28). It is also reported that curcumin effectively prevented emergence of chemoresistance and eliminate CSCs in breast, glioblastoma, pancreatic and colon cancer (29-33). The antiproliferation effects of curcumin on lung cancer have been reported to induce apoptosis in A549 and NCI-H460 cells through ER stress and caspase cascade- and mitochondriadependent pathways (34-36), however, the effects of curcumin on lung cancer stem-like cells remain obscure. In the present study, we investigated the underlying mechanisms of curcumin on lung tumor spheres.

\section{Materials and methods}

Cell culture and reagents. The lung cancer cell line NCI-H460 was purchased from American Type Culture Collection (ATCC) and cultured in RPMI-1640 supplemented with 
$10 \%$ fetal bovine serum (FBS). Curcumin and Taxol were purchased from Sigma. MTT was purchased from Amresco. The antibodies used in the present study were as follows: anti- $\beta$-actin (A5441; Sigma); p-JAK2 (\#3776), JAK2 (\#3230), p-STAT3 (\#9138), STAT3 (\#4904) (all from CST); C-myc (sc-40; Santa Cruz), anti-myc (\#MA1-21316-D680; Invitrogen), cyclin D1 (ab16663; Abcam). The growth factors used in the present study were: N2 supplement (Gibco), FGF, EGF (R\&D Systems), and STAT3 siRNA (\#6582; CST).

Cell viability and clonogenic assay. For cell viability assay, $8 \times 10^{4}$ cells were seeded into 12 -well plates. Then, different concentrations of curcumin and dimethyl sulfoxide (DMSO) were added into plates for indicated times. Viable cells were counted using trypan blue dye exclusion analysis. For the clonogenic assay, NCI-H460 cells were suspended in $1 \mathrm{ml}$ RPMI-1640 containing $0.3 \%$ low melting point agarose (Amresco) and 10\% FBS, and plated on a bottom layer containing $0.6 \%$ agarose and different doses of curcumin in $35 \mathrm{~mm}$ plates $(1,000$ cells/plate). Ten days later, cells were stained with $0.005 \%$ crystal violet and clones $>50$ cells were counted.

Tumor sphere assay. NCI-H460 cells were cultured in tumor sphere medium consisting of serum-free DMEM/F12 medium, $10 \mathrm{ng} / \mathrm{ml}$ human recombinant fibroblast growth factor-basic (FGF), $10 \mathrm{ng} / \mathrm{ml}$ epidermal growth factor (EGF), and N2 supplement. Then cells suspended in sphere medium containing different doses of curcumin and Taxol were plated into ultra low-attachment 24 well-plate at a density of 1,000/well. Five days later, tumor sphere formation was observed and photographed by a microscope.

Western blotting assay. NCI-H460 cells were suspended in tumor sphere medium to form tumor spheres. Then medium was replaced with fresh tumor sphere medium containing different doses of curcumin and DMSO and tumor spheres were cultured for indicated time points. Cell lysates of tumor spheres treated with curcumin or DMSO at indicated times or different doses were subjected to SDS-PAGE to detect related protein expression.

STAT3 overexpression and RNAi knockdown. STAT3 was overexpressed by transient transfection with pcDNA3.0-mycSTAT3 plasmids in H460 cells (vector pcDNA3.0-myc as control). To downregulate STAT3 expression in NCI-H460 cells, specific stat3 RNAi (CST) was used. After transfection, cells were suspended in tumor sphere medium in ultra low attachment 24-well plates. One week later, the formed tumor spheres were photographed under a microscope.

Murine models. All animal studies were conducted according to the protocols approved by the Animal Ethics Committee of Guangxi Medical University and maintained under conventional conditions. NCI-H460 derived tumor spheres were digested into single cells and injected into each mouse (100 cells for each) by subcutaneously inoculation in the right flank of nude mice. When tumor volume reached $50 \mathrm{~mm}^{3}$, mice were randomized into 3 groups ( $n=5$ for each group) and treated with curcumin $(40 \mathrm{mg} / \mathrm{kg})$, Taxol $(5 \mathrm{mg} / \mathrm{kg})$ or vehicle control (polyoxyethylenated castor oil: ethanol=1:1) for 2 weeks every 2 days with intraperitoneal injection (i.p). Tumor growth and mouse body weight were monitored every other day for 15 days. Tumor volume was calculated using the formula: $\mathrm{V}=0.5 \times \mathrm{L} \times \mathrm{W}^{2}$, where $\mathrm{L}$ and $\mathrm{W}$ represented the long and short diameter of the tumor, respectively. At the time of the animal sacrifice, tumors were excised; cells were separated and lyzed for western blotting.

Molecular docking. Computational docking test was performed using MOE2008.10 (Molecular Operating Environment) to investigate the interaction between JAK2 and curcumin at a molecular level. X-ray crystal structures of JAK2 (PDB ID: 5AEP) and its ligand was obtained from Protein Data Bank (http://www.rcsb.org). Water molecules were manually removed from the protein structures. Docking process was as described previously (39).

Statistical analysis. Differences between data groups were evaluated using Student's t-test. P-values $<0.05$ were considered to indicate a statistically significant result. Data are presented as the mean \pm SD unless otherwise noted.

\section{Results}

Curcumin inhibits proliferation and colony formation of NCI-H460 lung cancer cells. The effects of curcumin on lung cancer cell proliferation was determined using trypan blue dye exclusion analysis. The results demonstrated that curcumin significantly inhibited the growth of NCI-H460 cells at 20 and $40 \mu \mathrm{M}$ (Fig. 1B). The soft agar assay showed that curcumin markedly reduced colony number of NCI-H460 cells in a dosedependent manner (Fig. 1C and D). Curcumin significantly inhibited the colony forming activity of NCI-H460 cells at a concentration of $10 \mu \mathrm{M}$.

Curcumin impairs tumor sphere formation of NCI-H460 lung cancer cells in vitro. To determine the effects of curcumin on lung tumor spheres, we used NCI-H460 lung cancer cells as a in vitro model and carried out tumor sphere assays. The results suggested that curcumin reduced NCI-H460 tumor spheres in a dose-dependent manner (Fig. 2A). Compared with the traditional chemotherapy drug Taxol, which could not decrease tumor spheres (37), curcumin significantly reduced tumor spheres at concentrations of 20 and $40 \mu \mathrm{M}$ (Fig. 2B). These data implied curcumin suppressed tumor spheres growth in vitro.

Curcumin inhibits JAK2/STAT3 signaling pathway activity. Considering the significant role of JAK2/STAT3 signaling pathway in lung CSCs development $(23,38)$, we carried out western blotting to detect whether curcumin disturbed the JAK2/STAT3 signaling pathway. The results show that under curcumin treatment, p-JAK2 and p-STAT3 were downregulated in a time- and dose-dependent manner. The downstream molecules of the JAK2/STAT3 pathway, cyclin D1 and C-myc were also downregulated (Fig. 3A and B). Moreover, by molecular docking analysis (39), we discovered that curcumin could potently bind to JAK 2 . The three-dimensional structure revealed that curcumin can be perfectly embedded into the 
A<smiles>COc1cc(/C=C/C(=O)CC(=O)/C=C/c2ccc(O)cc2)ccc1O</smiles>

C

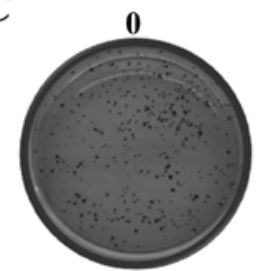

20

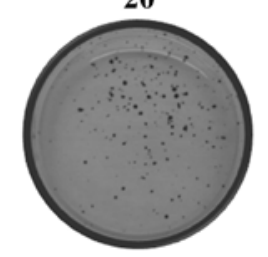

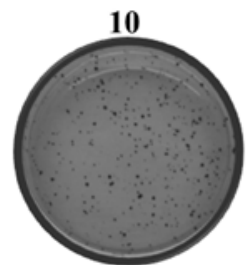

40

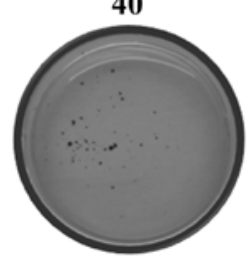

B

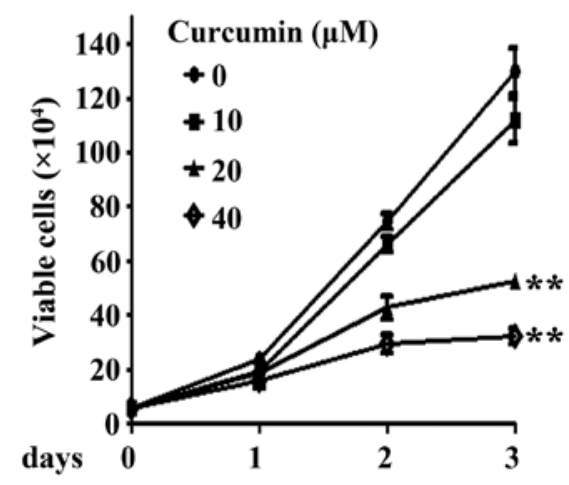

D

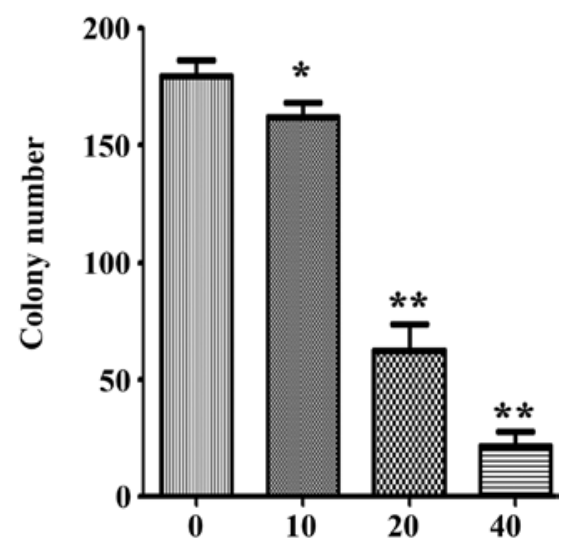

Figure 1. Curcumin inhibits proliferation and colony formation of NCI-H460 lung cancer cells. (A) Chemical structure of curcumin. (B) NCI-H460 cells were treated with curcumin for indicated time points and assessed by trypan blue exclusion analysis. (C) Soft-agar colony formation assay for NCI-H460 cells treated with or without curcumin. (D) Quantitation of foci counting. The data are presented as the mean \pm standard deviation with three independent experiments. ${ }^{*} \mathrm{P}<0.05,{ }^{* *} \mathrm{P}<0.01$, compared with the control group.

A

$\operatorname{Cur}(\mu \mathrm{M})$

DMSO

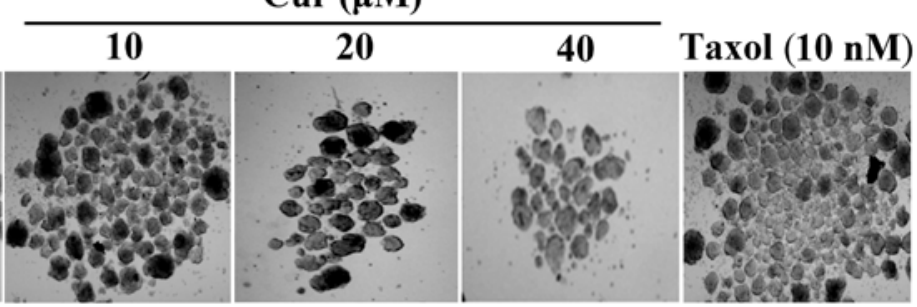

B

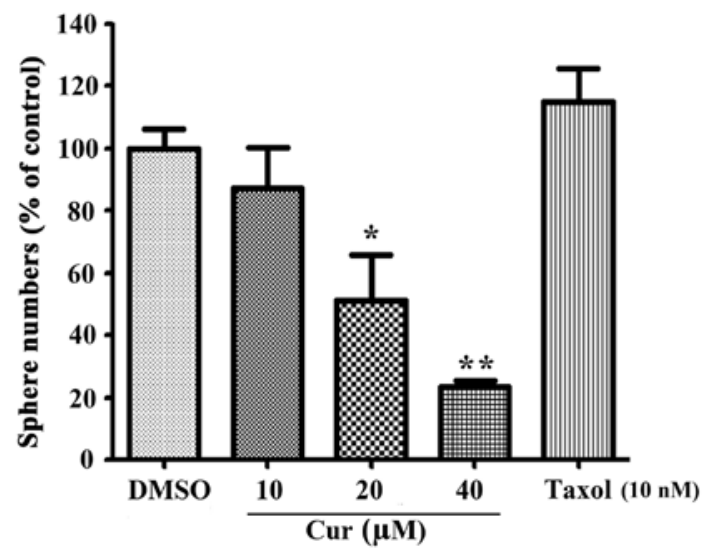

Figure 2. Curcumin impairs tumor sphere formation of NCI-H460 lung cancer cells. (A and B) NCI-H460 cells were plated into ultra low-attachment 24-well plates at a density of 1,000/well and cultured in tumor sphere medium containing different concentrations of curcumin and Taxol for 5 days. Tumor spheres were photographed (A) and tumor sphere numbers were counted using ImageJ (B). The data are presented as the mean \pm standard deviation with three independent experiments. ${ }^{*} \mathrm{P}<0.05,{ }^{* *} \mathrm{P}<0.01$, compared with the control group. 

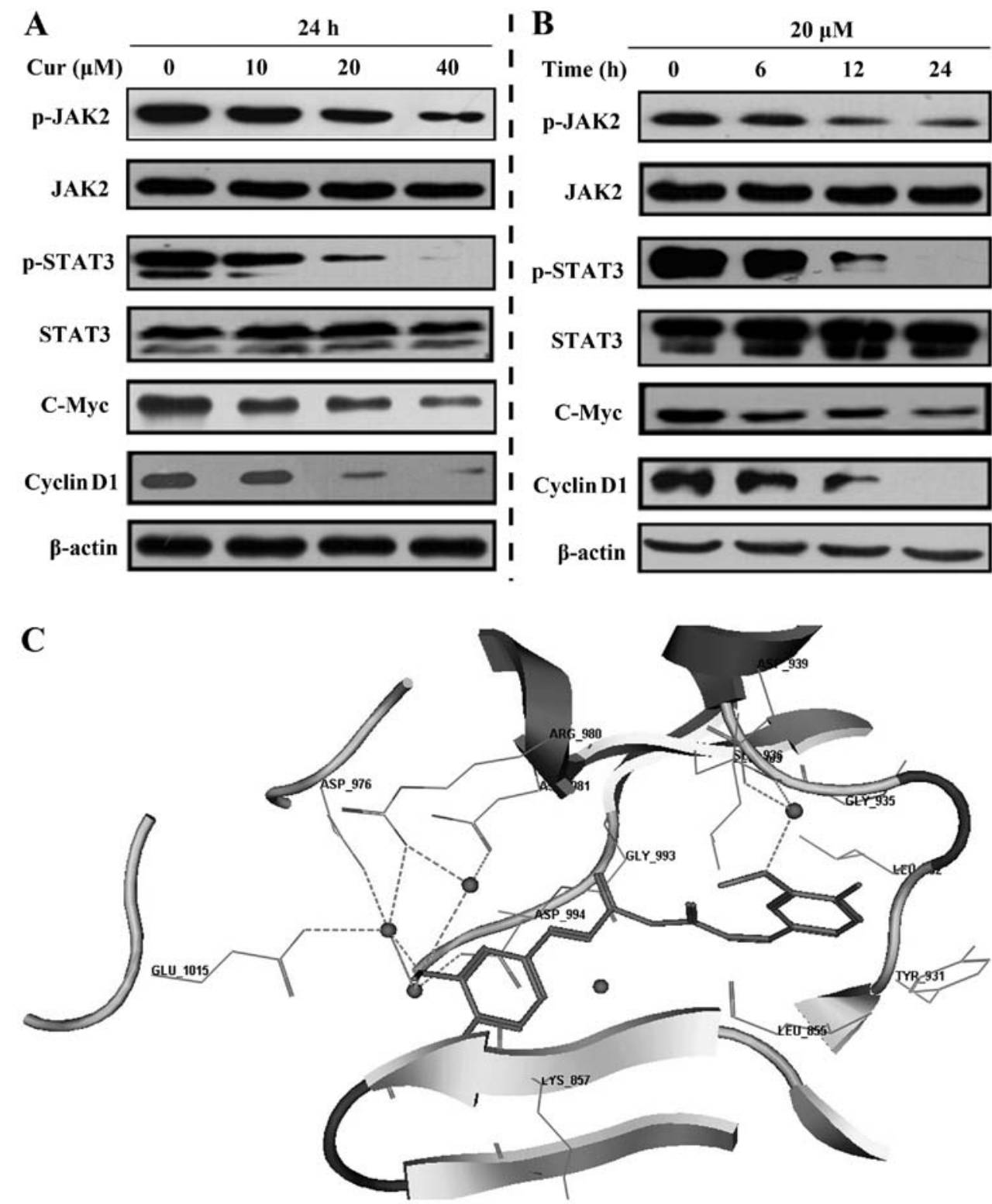

Figure 3. Curcumin inhibits JAK2/STAT3 signaling pathway activity. (A and B) Tumor spheres of NCI-H460 cells were treated with different doses of curcumin for $24 \mathrm{~h}$ (A) or $20 \mu \mathrm{m}$ curcumin for indicated times (B). Then western blotting assay was conducted using indicated antibodies. (C) Plausible binding model of curcumin to JAK2 is presented.

long and narrow hydrophobic pocket formed by Leu_855, Leu_983, Gly_858, Asp_994 and Gly_935 of JAK2 (Fig. 3C). The methoxyl group of curcumin formed hydrogen bonds with the side chains of Asp_976, Asp_994, Glu_1015,_Arg_980 and Asn_981 of JAK2 through water molecules (Fig. 3A). These results inferred that curcumin attenuated the JAK2/STAT3 pathway.

The role of STAT3 in curcumin-induced tumor sphere suppression. To further clarify JAK2/STAT3 activity in curcumin-induced tumor sphere suppression, we first evaluated STAT3 activity in NCI-H460 tumor sphere formation. STAT3 overexpressing and knockdown assays were carried out using plasmids and siRNA. We found when overexpressing STAT3, not only the number but the size of tumor spheres were increased (Fig. 4A). Conversely, when STAT3 was knoced down, both the number and size of tumor spheres were decreased (Fig. 4B). These results indicate that active stat 3 promotes tumor sphere formation of NCI-H460 lung cancer cells. Next, by stat 3 overexpression and curcumin co-treatment, stat 3 overexpression can restore curcumin-induced tumor sphere inhibition (Fig. 4C). Further, stat3 knockdown and curcumin treatment can synergistically inhibit tumor spheres of NCI-H460 lung cancer cells (Fig. 4D). Taken together, these results inferred curcumin could reduce tumor spheres via inhibiting the JAK2/STAT3 signaling pathway.

Curcumin inhibits tumor spheres growth of NCI-H460 lung cancer cells in vivo. We next assessed whether curcumin could inhibit stem-like tumor growth in vivo. NCI-H460 derived tumor spheres were digested into single cells and subcutaneously inoculated into nude mice in the right flank area (100 cells for each mouse). When the tumors reached $50 \mathrm{~mm}^{3}$, the mice were randomized into 3 groups $(\mathrm{n}=5$ for each group). The mice were administered curumin $(40 \mathrm{mg} / \mathrm{kg}$ ) or Taxol $(5 \mathrm{mg} / \mathrm{kg})$ every 2 days with intraperitoneal injection 
A

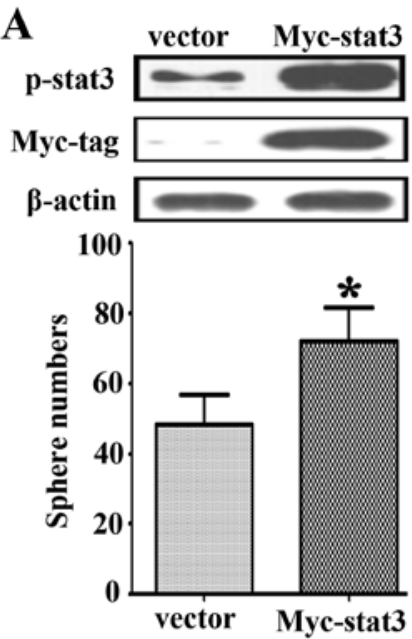

C

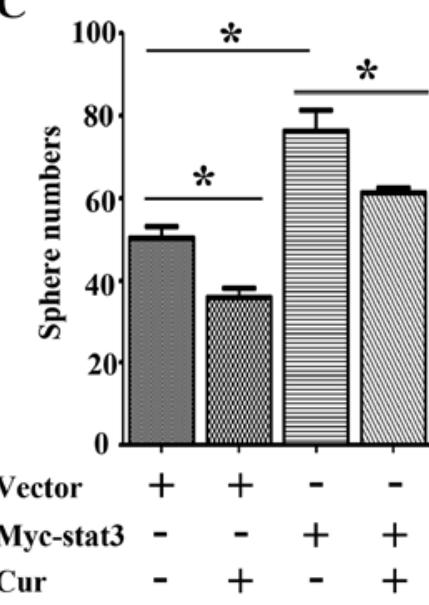

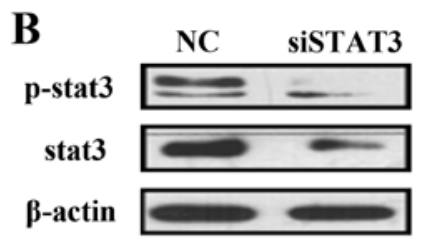

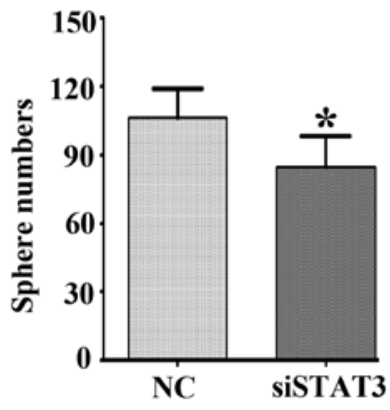

D

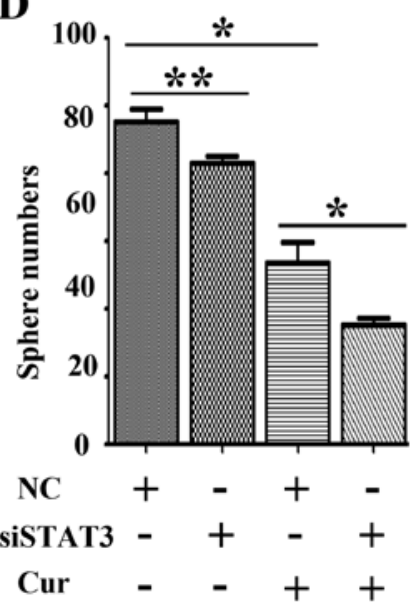

Figure 4. The role of STAT3 in curcumin induced NCI-H460 tumor sphere inhibition. (A and B) NCI-H460 cells of stat3 overexpression (A) or stat3 interference by siRNA (B) were seeded into ultra low-attachment 24-well plates at a density of 1,000/well. Five days later, tumor sphere numbers were counted and western blotting assay was conducted to detect p-STAT3 and STAT3 expression. (C and D) NCI-H460 cells of stat3 overexpression (C) or stat3 interference by siRNA (D) were treated with curcumin $(20 \mu \mathrm{M})$ for five days in tumor sphere medium and the number of tumor spheres was counted. The data are presented as the mean \pm standard deviation with three independent experiments. ${ }^{*} \mathrm{P}<0.05$, compared with the control group.

(i.p). Tumor growth and mice body weight were monitored every other day for 15 days. Intriguingly, we found that curcumin significantly inhibited tumor growth compared with vehicle control or Taxol $(\mathrm{P}<0.01)$ (Fig. 5A and $\mathrm{B})$. Moreover, body weight loss was not observed in curcumin-treated groups compared to vehicle and Taxol groups (Fig. 5C). Proteins were extracted from tumor samples and western blot assay was performed. We found that in curcumin treatment group, the expression of p-JAK2 and p-STAT3 were downregulated as compared with the samples separated from vehicle or Taxol control mice (Fig. 5D). These results demonstrate that curcumin suppressed tumor sphere growth in vivo.

\section{Discussion}

Tumor recurrence and drug resistance are the primary causes of poor survival rates in patients with advanced cancer. Since the isolation of CSCs in 1997, substantial research has demonstrated CSCs are highly related to tumor recurrence and drug resistance (12-14). Thus, eliminating CSCs may be a feasible approach for cancer therapy (40). Previous research showed that tumor spheres enrich cells with CSC-like property and are capable of forming tumors in vivo $(41,42)$. Unlike the main population of cancer cells, CSC-like cells can form tumor spheres in non-adherent conditions and serum-free medium (43). Tumor sphere-forming assay has its own advantage, as it does not rely on specific markers to identify CSCs, which could vary greatly from one cell line to another (44). Curcumin has been reported to be an effective drug by preventing emergence of chemoresistance and eliminating CSCs in breast, glioblastoma, pancreatic and colon cancer (29-33). To date, the effects and molecular mechanisms of curcumin on lung CSCs still remain unclear. In the present study, we performed tumor sphere assays to determine the effects of curcumin on lung CSCs and found curcumin impaired the ability of tumor spheres in NCI-H460 lung cancer cells (Fig. 2A and B). Moreover, in the in vivo nude mouse model, 100 cancer stem-like cells derived from NCI-H460 tumor spheres were injected into each mouse and tumors formed. Curcumin strongly repressed tumor growth compared to vehicle or Taxol treatment groups (Fig. 5A and B). These results indicated that curcumin suppressed tumor sphere growth of NCI-H460 lung cancer cells in vitro and in vivo.

Attempts to eliminate CSCs by targeting relevant signaling pathways are being carried out in several preclinical studies. In 


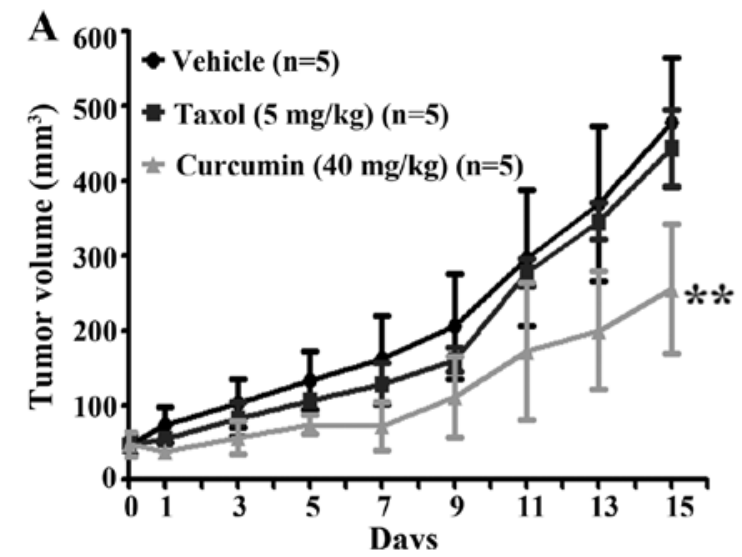

B

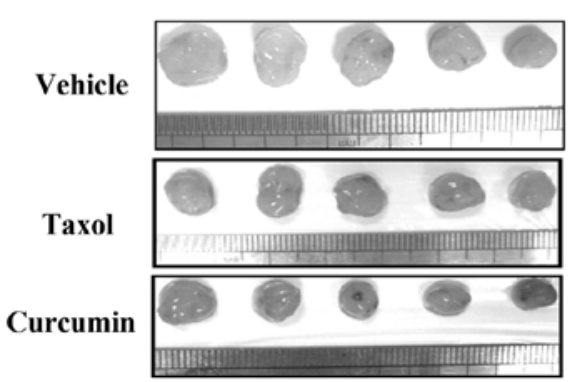

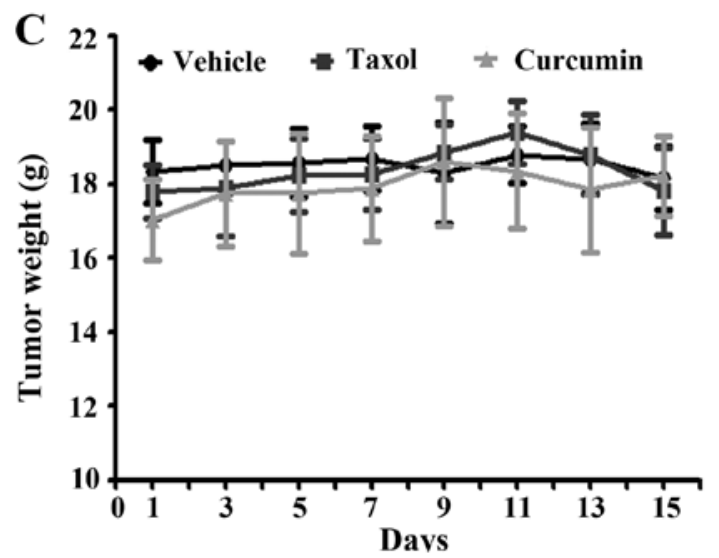

D

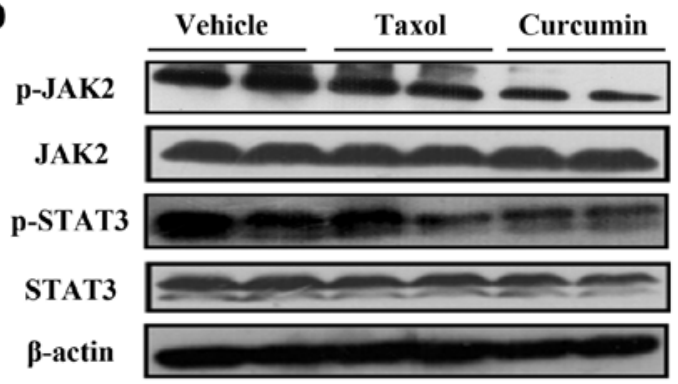

Figure 5. Curcumin inhibits tumor sphere derived tumor growth in xenograft mouse model. (A) Tumor volume was measured every two days and calculated according to the formula: $\mathrm{V}=0.5 \mathrm{x} \mathrm{L} \mathrm{x} \mathrm{W}$. (B) Images of xenograft tumors obtained from mice. (C) Body weight of mice was measured every two days. (D) Western blot analysis of lysates from tumor samples using indicated antibodies. The data are presented as the mean \pm standard deviation. ${ }^{* *} \mathrm{P}<0.01$, compared with the control group.

lung CSCs, interference with Wnt/ $\beta$-catenin signaling by RNAi markedly decreased cancer cell proliferation, clone formation and drug resistance (45). PTEN, Hedgehog, JAK-STAT, Notch and PI3K/AKT pathways also offer latent intervention targets against CSCs $(46,47)$. In the present study, by molecule docking we found curcumin interacted with the residues of JAK 2 by forming hydrogen bonds though water molecules (Fig. 3C). We assumed that curcumin could inhibit JAK2 activity and repress the JAK2/STAT3 signaling pathway. Western blotting results confirmed our hypothesis. Under curcumin treatment, the activity of JAK2/STAT3 pathway was inhibited in vitro and in vivo (Figs. 3A and $\mathrm{B}$, and $5 \mathrm{D}$ ). Further, to elucidate the role of STAT3 in tumor sphere formation of NCI-H460 lung cancer cells, we conducted an overexpressing and RNAi knockdown assay. By transfection with STAT3 overexpression plasmid or STAT3 siRNA, we found STAT3 activation promotes tumor sphere formation, while STAT3 inhibition suppresses this effect (Fig. 4A and B). Furthermore, by stat3 overexpression and curcumin co-treatment, stat 3 overexpression restored curcumin-induced tumor sphere inhibition, and stat 3 knockdown with curcumin could synergistically inhibit tumor sphere formatioin (Fig. 4C and D). Taken together, our results infer that curcumin repressed tumor spheres of NCI-H460 lung cancer cells by inhibiting the JAK2/STAT3 signaling pathway.

In conclusion, we have documented the antitumor sphere formation effects of curcumin in vitro and in vivo. Our findings highlighted the fact that curcumin could inhibit lung cancer cell proliferation, colony formation and tumor spheres. The underlying mechanisms of curcumin-induced tumor spheres suppression are mainly due to the inhibition of the
JAK2/STAT3 signaling pathway. The present study implies that curcumin may be a potential drug in lung CSC elimination and cancer therapy.

\section{Acknowledgements}

The present study was financially supported by the Natural Science Foundation of Zhejiang Province of China (no. Y12H300003), the Postdoctoral Science Foundation of China and the Postdoctoral Science Foundation of Guangxi Province of China. We thank Professor Hu Tingjun of School of Zoology Science and Technology of Guangxi University for the experimental support.

\section{References}

1. Jemal A, Bray F, Center MM, Ferlay J, Ward E and Forman D: Global cancer statistics. CA Cancer J Clin 61: 69-90, 2011.

2. Jordan CT, Guzman ML and Noble M: Cancer stem cells. N Engl J Med 355: 1253-1261, 2006.

3. Bonnet D and Dick JE: Human acute myeloid leukemia is organized as a hierarchy that originates from a primitive hematopoietic cell. Nat Med 3: 730-737, 1997.

4. Al-Hajj M, Wicha MS, Benito-Hernandez A, Morrison SJ and Clarke MF: Prospective identification of tumorigenic breast cancer cells. Proc Natl Acad Sci USA 100: 3983-3988, 2003.

5. Singh SK, Clarke ID, Terasaki M, Bonn VE, Hawkins C, Squire J and Dirks PB: Identification of a cancer stem cell in human brain tumors. Cancer Res 63: 5821-5828, 2003.

6. Miki J, Furusato B, Li H, Gu Y, Takahashi H, Egawa S, Sesterhenn IA, McLeod DG, Srivastava S and Rhim JS: Identification of putative stem cell markers, CD133 and CXCR4, in hTERT-immortalized primary nonmalignant and malignant tumor-derived human prostate epithelial cell lines and in prostate cancer specimens. Cancer Res 67: 3153-3161, 2007. 
7. Fang D, Nguyen TK, Leishear K, Finko R, Kulp AN, Hotz S, Van Belle PA, Xu X, Elder DE and Herlyn M: A tumorigenic subpopulation with stem cell properties in melanomas. Cancer Res 65: 9328-9337, 2005.

8. Ricci-Vitiani L, Lombardi DG, Pilozzi E, Biffoni M, Todaro M, Peschle $\mathrm{C}$ and De Maria R: Identification and expansion of human colon-cancer-initiating cells. Nature 445: 111-115, 2007.

9. O'Brien CA, Pollett A, Gallinger S and Dick JE: A human colon cancer cell capable of initiating tumour growth in immunodeficient mice. Nature 445: 106-110, 2007.

10. Kim CF, Jackson EL, Woolfenden AE, Lawrence S, Babar I, Vogel S, Crowley D, Bronson RT and Jacks T: Identification of bronchioalveolar stem cells in normal lung and lung cancer. Cell 121: 823-835, 2005.

11. Bertolini G, Roz L, Perego P, Tortoreto M, Fontanella E, Gatti L, Pratesi G, Fabbri A, Andriani F, Tinelli S, et al: Highly tumorigenic lung cancer $\mathrm{CD} 133^{+}$cells display stem-like features and are spared by cisplatin treatment. Proc Natl Acad Sci USA 106: 16281-16286, 2009.

12. Vinogradov $S$ and Wei $X$ : Cancer stem cells and drug resistance: The potential of nanomedicine. Nanomedicine 7: 597-615, 2012.

13. Chen K, Huang YH and Chen JL: Understanding and targeting cancer stem cells: Therapeutic implications and challenges. Acta Pharmacol Sin 34: 732-740, 2013.

14. Eyler CE and Rich JN: Survival of the fittest: Cancer stem cells in therapeutic resistance and angiogenesis. J Clin Oncol 26: 2839-2845, 2008.

15. Shigdar S, Lin J, Li Y, Yang CJ, Wei M, Zhus Y, Liu H and Duan W: Cancer stem cell targeting: The next generation of cancer therapy and molecular imaging. Ther Deliv 3: 227-244, 2012.

16. Sansone $\mathrm{P}$ and Bromberg J: Targeting the interleukin-6/Jak/stat pathway in human malignancies. J Clin Oncol 30: 1005-1014, 2012.

17. Sun Y, Moretti L, Giacalone NJ, Schleicher S, Speirs CK Carbone DP and Lu B: Inhibition of JAK2 signaling by TG101209 enhances radiotherapy in lung cancer models. J Thorac Oncol 6: 699-706, 2011.

18. Colomiere M, Ward AC, Riley C, Trenerry MK, CameronSmith D, Findlay J, Ackland L and Ahmed N: Cross talk of signals between EGFR and IL-6R through JAK2/STAT3 mediate epithelial-mesenchymal transition in ovarian carcinomas. Br J Cancer 100: 134-144, 2009.

19. Behera R, Kumar V, Lohite K, Karnik S and Kundu GC: Activation of JAK2/STAT3 signaling by osteopontin promotes tumor growth in human breast cancer cells. Carcinogenesis 31 $192-200,2010$

20. Zhao M, Gao FH, Wang JY, Liu F, Yuan HH, Zhang WY and Jiang B: JAK2/STAT3 signaling pathway activation mediates tumor angiogenesis by upregulation of VEGF and bFGF in nonsmall-cell lung cancer. Lung Cancer 73: 366-374, 2011.

21. Tan DS, Agarwal R and Kaye SB: Mechanisms of transcoelomic metastasis in ovarian cancer. Lancet Oncol 7: 925-934, 2006.

22. Abubaker K, Luwor RB, Zhu H, McNally O, Quinn MA, Burns CJ, Thompson EW, Findlay JK and Ahmed N: Inhibition of the JAK2/STAT3 pathway in ovarian cancer results in the loss of cancer stem cell-like characteristics and a reduced tumor burden. BMC Cancer 14: 317, 2014.

23. Hsu HS, Lin JH, Hsu TW, Su K, Wang CW, Yang KY, Chiou SH and Hung SC: Mesenchymal stem cells enhance lung cancer initiation through activation of IL-6/JAK2/STAT3 pathway. Lung Cancer 75: 167-177, 2012.

24. Marotta LL, Almendro V, Marusyk A, Shipitsin M, Schemme J, Walker SR, Bloushtain-Qimron N, Kim JJ, Choudhury SA, Maruyama R, et al: The JAK2/STAT3 signaling pathway is required for growth of $\mathrm{CD} 44^{+} \mathrm{CD} 24^{-}$stem cell-like breast cancer cells in human tumors. J Clin Invest 121: 2723-2735, 2011.

25. Chauhan DP: Chemotherapeutic potential of curcumin for colorectal cancer. Curr Pharm Des 8: 1695-1706, 2002.

26. Leu TH and Maa MC: The molecular mechanisms for the antitumorigenic effect of curcumin. Curr Med Chem Anticancer Agents 2: 357-370, 2002.

27. Karunagaran D, Rashmi R and Kumar TR: Induction of apoptosis by curcumin and its implications for cancer therapy. Curr Cancer Drug Targets 5: 117-129, 2005.

28. Duvoix A, Blasius R, Delhalle S, Schnekenburger M, Morceau F, Henry E, Dicato $M$ and Diederich M: Chemopreventive and therapeutic effects of curcumin. Cancer Lett 223: 181-190, 2005.
29. Fong D, Yeh A, Naftalovich R, Choi TH and Chan MM Curcumin inhibits the side population (SP) phenotype of the rat C6 glioma cell line: Towards targeting of cancer stem cells with phytochemicals. Cancer Lett 293: 65-72, 2010.

30. Kakarala M, Brenner DE, Korkaya H, Cheng C, Tazi K, Ginestier C, Liu S, Dontu G and Wicha MS: Targeting breast stem cells with the cancer preventive compounds curcumin and piperine. Breast Cancer Res Treat 122: 777-785, 2010.

31. Lim KJ, Bisht S, Bar EE, Maitra A and Eberhart CG: A polymeric nanoparticle formulation of curcumin inhibits growth, clonogenicity and stem-like fraction in malignant brain tumors. Cancer Biol Ther 11: 464-473, 2011.

32. Lin L, Liu Y, Li H, Li PK, Fuchs J, Shibata H, Iwabuchi Y and Lin J: Targeting colon cancer stem cells using a new curcumin analogue, GO-Y030. Br J Cancer 105: 212-220, 2011.

33. Bao B, Ali S, Banerjee S, Wang Z, Logna F, Azmi AS, Kong D, Ahmad A, Li Y, Padhye S, et al: Curcumin analogue CDF inhibits pancreatic tumor growth by switching on suppressor microRNAs and attenuating EZH2 expression. Cancer Res 72: 335-345, 2012.

34. Radhakrishna Pillai G, Srivastava AS, Hassanein TI, Chauhan DP and Carrier E: Induction of apoptosis in human lung cancer cells by curcumin. Cancer Lett 208: 163-170, 2004.

35. Lin SS, Huang HP, Yang JS, Wu JY, Hsia TC, Lin CC, Lin CW, Kuo CL, Gibson Wood W and Chung JG: DNA damage and endoplasmic reticulum stress mediated curcumin-induced cell cycle arrest and apoptosis in human lung carcinoma A-549 cells through the activation caspases cascade- and mitochondrialdependent pathway. Cancer Lett 272: 77-90, 2008.

36. Wu SH, Hang LW, Yang JS, Chen HY, Lin HY, Chiang JH, Lu CC, Yang JL, Lai TY, Ko YC, et al: Curcumin induces apoptosis in human non-small cell lung cancer NCI-H460 cells through ER stress and caspase cascade- and mitochondria-dependent pathways. Anticancer Res 30: 2125-2133, 2010.

37. Gupta PB, Onder TT, Jiang G, Tao K, Kuperwasser C, Weinberg RA and Lander ES: Identification of selective inhibitors of cancer stem cells by high-throughput screening. Cell 138: 645-659, 2009

38. Shao C, Sullivan JP, Girard L, Augustyn A, Yenerall P, Rodriguez-Canales J, Liu H, Behrens C, Shay JW, Wistuba II, et al: Essential role of aldehyde dehydrogenase $1 \mathrm{~A} 3$ for the maintenance of non-small cell lung cancer stem cells is associated with the STAT3 pathway. Clin Cancer Res 20: 4154-4166, 2014.

39. Padilla F, Bhagirath N, Chen S, Chiao E, Goldstein DM, Hermann JC, Hsu J, Kennedy-Smith JJ, Kuglstatter A, Liao C, et al: Pyrrolopyrazines as selective spleen tyrosine kinase inhibitors. J Med Chem 56: 1677-1692, 2013.

40. Li RJ, Ying X, Zhang Y, Ju RJ, Wang XX, Yao HJ, Men Y, Tian W, Yu Y, Zhang L, et al: All-trans retinoic acid stealth liposomes prevent the relapse of breast cancer arising from the cancer stem cells. J Control Release 149: 281-291, 2011.

41. Grimshaw MJ, Cooper L, Papazisis K, Coleman JA, Bohnenkamp HR, Chiapero-Stanke L, Taylor-Papadimitriou J and Burchell JM: Mammosphere culture of metastatic breast cancer cells enriches for tumorigenic breast cancer cells. Breast Cancer Res 10: R52, 2008.

42. Ponti D, Costa A, Zaffaroni N, Pratesi G, Petrangolini G, Coradini D, Pilotti S, Pierotti MA and Daidone MG: Isolation and in vitro propagation of tumorigenic breast cancer cells with stem/progenitor cell properties. Cancer Res 65: 5506-5511, 2005.

43. Cioce M, Gherardi S, Viglietto G, Strano S, Blandino G, Muti P and Ciliberto G: Mammosphere-forming cells from breast cancer cell lines as a tool for the identification of CSC-like- and early progenitor-targeting drugs. Cell Cycle 9: 2878-2887, 2010.

44. Park SY, Lee HE, Li H, Shipitsin M, Gelman R and Polyak K: Heterogeneity for stem cell-related markers according to tumor subtype and histologic stage in breast cancer. Clin Cancer Res 16: 876-887, 2010.

45. Teng Y, Wang X, Wang Y and Ma D: Wnt/beta-catenin signaling regulates cancer stem cells in lung cancer A549 cells. Biochem Biophys Res Commun 392: 373-379, 2010.

46. Yilmaz OH, Valdez R, Theisen BK, Guo W, Ferguson DO, Wu H and Morrison SJ: Pten dependence distinguishes haematopoietic stem cells from leukaemia-initiating cells. Nature 441: 475-482, 2006.

47. Lin L, Fuchs J, Li C, Olson V, Bekaii-Saab T and Lin J: STAT3 signaling pathway is necessary for cell survival and tumorsphere forming capacity in $\mathrm{ALDH}^{+} / \mathrm{CD} 133^{+}$stem cell-like human colon cancer cells. Biochem Biophys Res Commun 416: 246-251, 2011. 